\title{
Triggered massive star formation in the vicinity of WR 48a
}

\author{
J. S. Clark ${ }^{1}$ and J. M. Porter ${ }^{2}$ \\ ${ }^{1}$ Department of Physics and Astronomy, University College London, Gower Street, London, WC1E 6BT, England, UK \\ e-mail: jsc@star.ucl.ac.uk \\ 2 Astrophysics Research Institute, Liverpool John Moores University, Twelve Quays House, Egerton Wharf, Birkenhead, \\ CH41 1LD, UK
}

Received 25 March 2004 / Accepted 23 July 2004

\begin{abstract}
We utilise Midcourse Space Experiment mid-IR imaging and published data to discuss the (massive) star formation region at galactic longitude $\sim 305^{\circ}$, apparently associated with the Wolf Rayet WR $48 \mathrm{a}$ and the attendant clusters Danks 1 and 2. A spectacular three lobed wind blown bubble surrounds the aforementioned sources, for which we may infer a minimum age of $\sim 3$ Myr from the presence of the WCL star. Near IR data reveals the presence of numerous embedded sources on the periphery of the wind blown bubble. The presence of coincindent $\mathrm{H}_{2} \mathrm{O}, \mathrm{OH}$ and methanol maser emission is suggestive of ongoing massive star formation, which is suppported by the fluxes of the associated IR sources, and the requisite LyC flux required to support the emission from the subset that have associated ucH II regions. Consideration of the integrated radio flux of the complex implies that a minimum of $31 \mathrm{O} 7 \mathrm{~V}$ stars must be present, under the assumption of no photon leakage. Given the age and morphology of the complex and in particular the observation that the central exciting clusters have entirely cleared their natal material, we expect this assumption will be violated, and hence that the true population of massive stars is likely to be significantly larger. If confirmed, the G305 complex represents one of the most massive regions of ongoing triggered star formation currently identified in the galaxy.
\end{abstract}

Key words. stars: Wolf-Rayet - stars: individual: WR 48a - stars: formation - ISM: H II regions

\section{Introduction}

As significant sources of ionising radiation, mechanical energy and chemically enriched material, massive OB stars play a crucial role in the wider galactic ecology. However, these properties also distinguish massive-star formation mechanisms from a simple "scaled up" version of low-mass star formation; their powerful winds and radiation fields can act to both inhibit accretion onto the massive protostar, whilst disrupting their larger natal environs. This activity has been proposed to trigger (e.g. Elmegreen \& Lada 1977) or alternatively inhibit subsequent generations of star formation via the compression or dispersal, respectively, of natal molecular clouds.

Observationally, investigation of massive-star birth is hampered by the comparative rarity of $\mathrm{OB}$ stars, compounded by the rapidity assumed for the formation process $\left(10^{5} \mathrm{yr}\right.$, e.g. McKee \& Tan 2002) and the significant extinction evident to many sources. As part of a wider programme of research into early-type stars and their environs, we have utilised the Midcourse Space Experiment (MSX) Galactic Plane Survey (GPS) to investigate the mid-IR properties of the galactic population of Wolf Rayets (WR; Clark \& Porter, in prep.). Given the youth and high mass of such objects it was hoped that they might serve as signposts for regions of recent $(<10 \mathrm{Myr})$ massive star formation. Here we report on one such example the region around WR 48a.

WR 48a is a dusty WCL star (Danks et al. 1983) found to be within $\sim 1^{\prime}$ of the two heavily reddened, optically visible clusters Danks 1 and 2 - which are themselves separated by only $\leq 2^{\prime}$. This suggests an origin of WR 48a in one or other is highly likely (Danks et al. 1984). With the exception of the $\mathrm{H} \alpha$ nebula RCW 74, the heavy foreground extinction $\left(A_{\mathrm{v}}=9 \mathrm{mag}\right.$ for WR 48a) prevents the optical detection of the numerous H II regions detected at radio wavelengths within the $\sim 1.5^{\circ} \times 1.5^{\circ}$ region centred on WR 48a (e.g. Goss \& Shaver 1970; Danks et al. 1984). These have been suggested to comprise a single extended region of active star formation designated as G305 by Georgelin et al. (1988). Despite the significance of this hypothesis - the integrated radio luminosity would be directly comparable to the most luminous giant H II regions of our galaxy - the putative complex has globally remained unstudied for the past 2 decades.

In this paper we present mid-IR images that support Georgelin et al.'s hypothesis and combine existing near-IR to radio observations to demonstrate the occurence of ongoing, sequential massive star formation triggered by WR 48a and the stellar populations of Danks 1 and 2. Our criteria for the morphological signatures of triggered star formation are those suggested by Elmegreen (1992): (i) two stellar populations 
differing in age by several Myr and with separations of $\sim 10-50 \mathrm{pc}$ and (ii) a substantial amount of gas near the younger subgroup with little or no gas associated with the older subgroup (Elmegreen also suggested a third signature, a velocity difference between the older and younger subgroup of $\sim 5-$ $10 \mathrm{~km} \mathrm{~s}^{-1}$, although we cannot test for this with the current datasets).

In Sect. 2 we describe the MSX observations of the region surrounding WR 48a, and in Sect. 3 we discuss the multiwavelength evidence for recent star formation close to (associated with) WR 48a. We present a Discussion in Sect. 4 before our Conclusions in Sect. 5.

\section{MSX mid-IR data}

Mill et al. (1994) give an overview of MSX mission and instruments, while a detailed account of the characteristics of the infrared telescope and the GPS can be found in Price et al. (2001). In Fig. 1 we present a false colour image ${ }^{1}$ of the MSX in-band radiance $\left(\mathrm{W} \mathrm{m}^{-2} \mathrm{sr}^{-1}\right)$ of the $1.5^{\circ} \times 1.5^{\circ}$ region centred on $\mathrm{WR}$ $48 \mathrm{a}$, where band $A(8.3 \mu \mathrm{m})$ is encoded in blue, the sum of bands $C$ and $D(12.1 \mu \mathrm{m}$ and $14.7 \mu \mathrm{m}$ respectively) radiance is encoded as green, and band $E(21.3 \mu \mathrm{m})$ is red.

In Fig. 2 we plot the band $A$ data and overplot the positions of WR 48a, the optically visible clusters Danks 1 and 2, as well as the positions of the newly discovered cluster candidates [DBS2003] 82-84 and 130-134 (discovery and notation given by Dutra et al. 2003), associated $\mathrm{H}$ II regions, $\mathrm{CH}_{3} \mathrm{OH}$ maser emission and IRAS sources with colours consistent with ucH II regions, as described in the figure caption and Sect. 3 .

Figure 2 dramatically shows the intense activity occuring within $\sim 0.7^{\circ}(\leq 34 \mathrm{pc}$ at a distance of $4 \mathrm{kpc}$; see Sect. 4 for justification) of both WR 48a and Danks 1 and 2. We identify three distinct lobes of emission, with maximal projected extents relative to WR 48a of $\sim 32 \mathrm{pc}$ (galactic NE lobe), $\sim 19$ pc (galactic $\mathrm{NW}$ lobe) and $\sim 34 \mathrm{pc}$ (galactic $\mathrm{S}$ lobe). Of these the sizes of the first two lobes were measured from the well defined band $A$ boundaries. For the southern lobe we measured the extent from band $E$ data, given the lack of a defined lobe in band $A$, where a complex structure of nested filamentary emission dominates instead (Fig. 2). Nevertheless, we find a faint, continuous arc of emission in the band $D$ and $E$ images connecting both NE and southern lobes. Given the location of Danks 1 and 2 - both within $\sim 4^{\prime}$ of the cusp of this structure - we suspect that the emission arises from a cool dusty component entrained in a wind driven by stars in one or both clusters.

The main body of the nebula is delineated by a highly complex structure of scalloped filaments in band $A$, which are particularly evident in the easterly edge of the NE lobe (Fig. 2). At longer wavelengths the emission is smoother, although numerous (spatially extended) "hot spots" are present and spatially coincident in all four wavebands. These have colour

\footnotetext{
1 Individual image plates were created by convolving the full data set of survey scans with a 7.06" FWHM Gaussian onto the image grid, which has $6^{\prime \prime}$ centers. This pixel spacing is the smallest that the data sampling can support, given the measured point response function and the system modulation transfer function; the resultant image products have a resolution of $\sim 20^{\prime \prime}$.
}

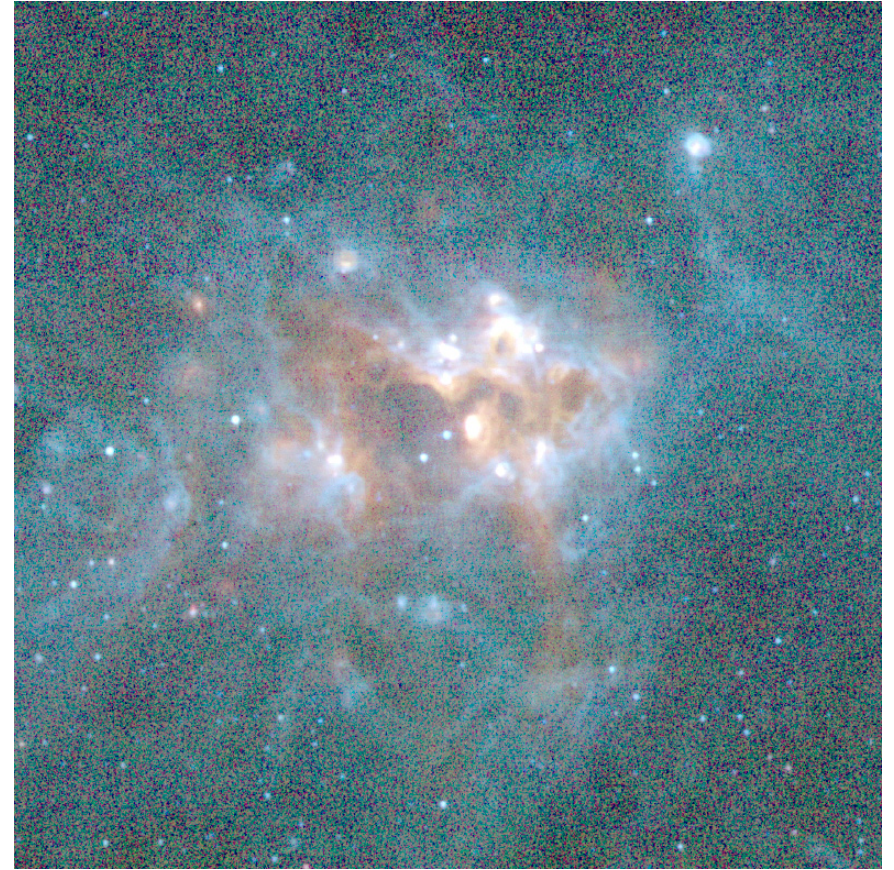

Fig. 1. 3 colour image of the $1.5^{\circ} \times 1.5^{\circ}$ field centred on WR $48 \mathrm{a}$ and Danks 1 and 2. Blue: band $A(6.8-10.8 \mu \mathrm{m})$; green: bands $C$ and $D$ (11.1-15.9 $\mu \mathrm{m})$; red: band $E(18.2-25.1 \mu \mathrm{m})$.

temperatures of $\sim$ hundred Kelvin and are likely to correspond to regions of embedded star formation (Sect. 3).

We find that the peak in the longer wavelength band $D$ and $E$ emission to be displaced interior to the shorter wavelength band $A$ emission. The same behaviour is reported for the Rosetta nebula studied by Kraemer et al. (2003), who propose that the dominant sources of emission at long and short wavelengths are warm dust and PAH molecules, respectively. Therefore, by analogy to this object we suggest that the striation represents a photodissociation front expanding into the parent molecular clouds with heated dust found interior to this.

The NE and S lobes are extremely reminiscent of an elongated wind blown bubble, as is the NW lobe (particularly visible in the band $A$ emission Fig. 2). The region within these boundaries has little emission indicative of an evacuated region. It is unlikely that patchy absorption would be able to provide such a coherent structure on the plane of the sky, and so we associate the lobes with cavities.

At the centre of these two overlapping cavities are the clusters Danks 1 and 2 and WR 48a (Fig. 2). Neither Danks 1 or 2 are visible in the MSX data - at the distance of the complex (see Sect. 4), it is likely that we could have only detected red supergiants or hot stars associated with a significant IR excess (similar to the case of Westerlund 1, Clark et al. 2004). WR 48a is present and visible as the bright blue-ish object in the centre of the field; the emission is due to its dusty circumstellar environment (=MSX6C G305.3614+00.0561; Clark \& Porter 2004, in prep.). A similarly luminous point source MSX6C G305.4013+00.0170 - is located to the south east of WR 48a. No optical counterpart is reported in the literature although the $J H K$ photometry for this source from the 2MASS survey show an emission falloff indicative of an object with 


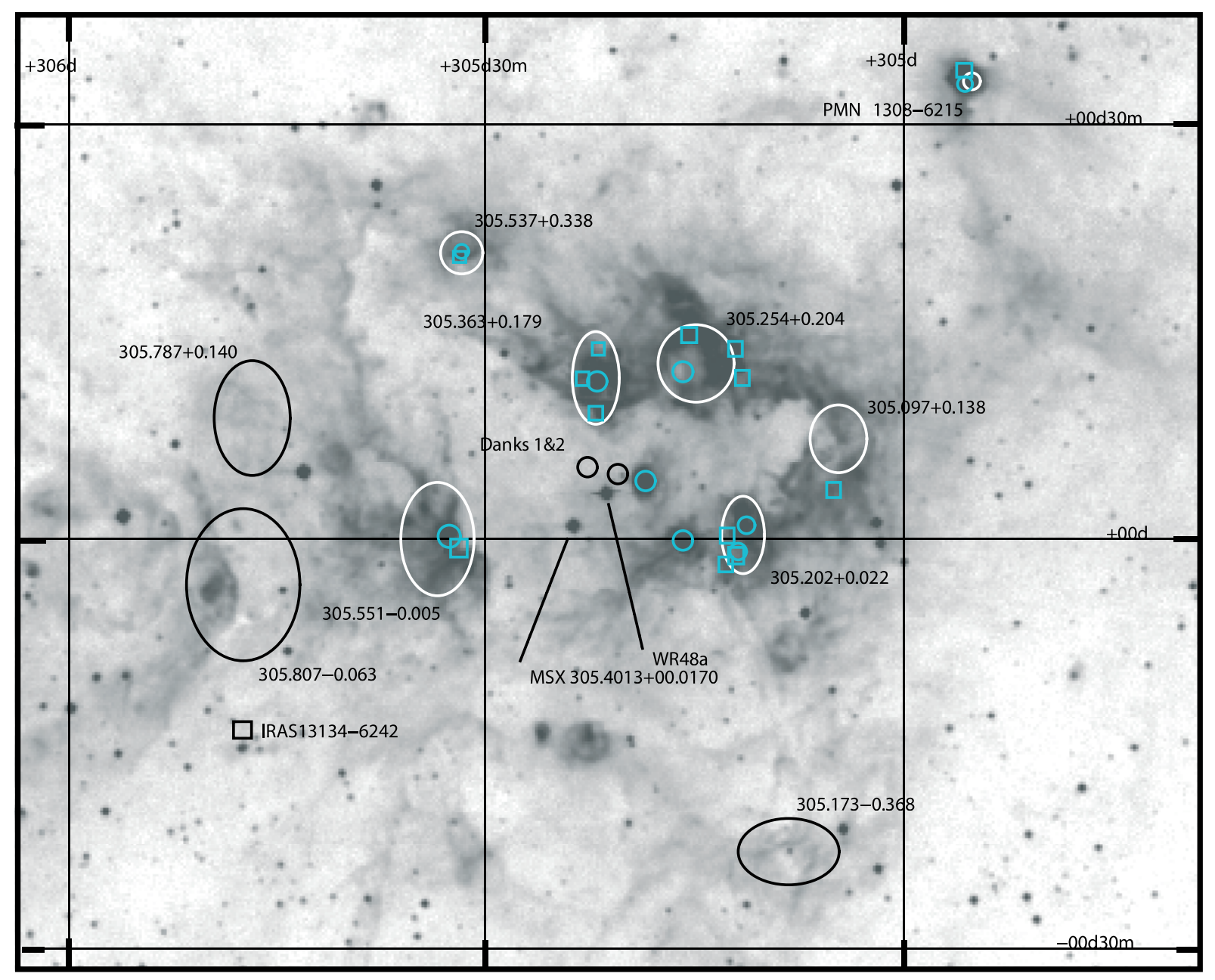

Fig. 2. Greyscale plot of the band $A$ data for the field presented in Fig. 1. Positions of H II regions are shown semi schematically, using the dimensions given in Caswell \& Haynes (1987), determined from the FWHM of Gaussian fits to the sources. See Fig. 2 of Danks et al. (1984) for a complimentary map of $20 \mathrm{~cm}$ emission for the inner regions of the complex, which is clearly indicative of emission extending beyond the ellipses plotted here. The positions of the near-IR cluster candidates detailed in Sect. 3.1 are given by the cyan circles, while the IRAS point sources and maser emission listed in Table 2 are given by the cyan squares (with the exception of IRAS 13134-6242, which is presented in black for clarity in grayscale versions of this figure). Note that the radio source PMN J1308-6215 is unresolved and just the position is indicated by the symbol, while the radio map of Danks et al. (1984) suggests radio emission may be associated with the clusters [DBS2003] 130 and 132, which do not have corresponding Caswell \& Haynes (1987) detections.

high extinction $\left(A_{\mathrm{V}}>10 \mathrm{mag}\right)$. With fluxes of 53.2, 54.7, 42.0 and $47.8 \mathrm{Jy}$ for bands $A, C, D$ and $E$ respectively, MSX6C G305.4013+00.0170 has IR colours consistent with an identification as a dusty WCL star (Clark \& Porter, 2004, in prep.), although without further data we cannot make a definitive statement on its nature ${ }^{2}$. We therefore suggest that WR 48a and the hot star components of the clusters Danks 1 and 2 are the driving sources for the cavities.

${ }^{2}$ We note that the mid-IR colours of MSX6C G305.4013+00.0170 differ from those of WR 48a, although they both lie within the range occupied by dusty WCL stars. Dusty WCL stars are thought to reside in massive binary systems (e.g. Williams \& van der Hucht 2000), with the dust forming in wind collision zones. Hence the spread in midIR colours likely results from different binary parameters, which yield different dust productions rates - continuous or episodic - and hence temperatures.
Finally, we identify two compact bubble like structures $\left(\sim 2^{\prime}\right.$ in diameter; coincident with PMN J1308-6215 and [CH87] $305.537+0.338$ in Fig. 2 and Table 2) which are of interest, since their emission mirrors the nested nature of the main nebula. We speculate that these too host young exciting sources that are beginning to interact with their environs.

\section{Star formation tracers}

In order to elucidate the properties of the putative MSX wind blown nebula we performed a literature search for evidence for embedded sources and associated molecular, maser and $\mathrm{H} \mathrm{II}$ emission to identify possible ongoing star formation. The results of this search show significant activity in a number of different regions distributed over the field as indicated in Fig. 2. 
Table 1. Summary of H II regions comprising the star forming complex from Caswell \& Haynes (1987; $\lambda=6 \mathrm{~cm}$, upper panel) and Danks et al. (1984; $\lambda=20 \mathrm{~cm}$, lower panel). Radio fluxes, requisite LyC ionising fluxes and the corresponding number of canonical O7V stars are listed. Finally, associated IR clusters and cluster candidates from Dutra et al. (2003) are listed, as well as the anonymous IR cluster associated with [CH87] 305.363+0.179 (Conti \& Crowther 2004). Positional uncertainty for the H II regions is only 1 arcsec (Caswell \& Haynes 1987); thus given the $\sim$ few arcmin extent of the H II regions, we are confident of positional coincidence with the Dutra et al. (2003) cluster sample.

\begin{tabular}{lcccl}
\hline \hline Radio Source & $\begin{array}{c}\text { Flux } \\
\text { (Jy) }\end{array}$ & $\begin{array}{c}\log N \\
\text { LyC }\end{array}$ & $\begin{array}{c}\text { No. } \\
\text { O7V }\end{array}$ & $\begin{array}{l}\text { Associated } \\
\text { Source(s) }\end{array}$ \\
\hline [CH87] 305.097+0.138 & 15.3 & 49.37 & 2 & - \\
[CH87] 305.173-0.368 & 10.0 & 49.20 & 2 & - \\
[CH87] 305.202+0.022 & 26.0 & 49.60 & 4 & [DBS2003] 83 and 84 \\
[CH87] 305.254+0.204 & 51.0 & 49.90 & 8 & [DBS2003] 131 \\
[CH87] 305.363+0.179 & 37.3 & 49.76 & 6 & Anonymous IR cluster \\
[CH87] 305.537+0.338 & 5.4 & 48.92 & 1 & [DBS2003] 133 \\
[CH87] 305.551-0.005 & 35.4 & 49.74 & 6 & [DBS2003] 134 \\
[CH87] 305.787+0.140 & 5.7 & 48.94 & 1 & - \\
[CH87] 305.807-0.063 & 6.5 & 49.00 & 1 & - \\
PMN J1308-6215 & 0.5 & 47.90 & O9V & [DBS2003] 82 \\
Complex Total (6 cm) & 193.1 & 50.50 & 31 & \\
& & & & \\
Danks84 G305.27-0.01 (20 cm) & 8.4 & 49.02 & 1 & [DBS2003] 130 \\
Danks84 G305.32+0.07 (20 cm) & 6.3 & 48.90 & 1 & [DBS2003] 132 \\
\hline
\end{tabular}

\subsection{Near IR emission and embedded clusters}

Star formation frequently proceeds in clustered environs (e.g. the review of Clarke et al. 2000), and so we have utilised the analysis of the 2MASS dataset of Dutra et al. (2003) to identify embedded clusters in the complex.

Dutra et al. (2003) claim to identify 5 star clusters in addition to Danks 1 and 2 - within the confines of the complex, denoted [DBS2003] 130, 131, 132, 133 and 134. Of these, [DBS2003] 130, $133 \& 134$ are coincident with mid-IR hotspots on the periphery of the wind blown bubble. [DBS2003] 131 sits in a circular $\left(d \sim 2.6^{\prime}\right)$ void bounded by strong emission - particularly to the NW - and [DBS2003] 132 within the westerly wind blown lobe, situated at the apex of the faint arc of emission visible in bands $D$ and $E$ at a projected distance of only $\sim 2 \mathrm{pc}$ from Danks 1.

Unfortunately, the compact nature of the putative clusters prevents the construction of individual HR diagrams from the present 2MASS dataset - clearly observations with a higher spatial resolution will be required to accomplish this.

Furthermore, adopting Dutra et al.'s notation, the IR grouping [DBS2003] 82 is associated with the MSX bubble source/H II region PMN J1308-6215 (Table 1), while the cluster candidates [DBS2003] 83 and 84 are coincident with the radio source [CH87] 305.202+0.022 (see Sect. 3.3). However, high resolution $K$ band images of this region by Walsh et al. (2001) apparently reveal the extended emission to be nebular in origin.

Finally, although not identified as a cluster we note that unpublished data for the vicinity of [CH87] $305.363+0.179$ reveals a further cluster not visible in the 2MASS dataset due to the presence of a nearby bright field star (R. Blum, 2004, priv. comm.; Conti \& Crowther 2004).

\subsection{H II regions}

Significant H II emission has been observed in the field by several authors, although systematic analysis is complicated by the different beam sizes used and wavelengths observed, which renders the cross correlation of individual sources problematic. For simplicity we have therefore restricted ourselves to the $6 \mathrm{~cm}$ survey of Caswell \& Haynes (1987) and also the $20 \mathrm{~cm}$ pointed observations of Danks (1984). We find nine H II regions in the Caswell \& Haynes collation and an additional two radio sources from Danks' study, which we list in Table 1.

Caswell \& Haynes (1987) only provide sizes for the individual $\mathrm{H}$ II regions, but overplotting the resultant ellipses on the band $A$ emission (Fig. 2) and comparison to the $20 \mathrm{~cm}$ map of Danks et al. (1984) reveals an obvious correspondance between the location of H II emission and the morphology of the MSX mid-IR nebulosity. The H II emission clearly traces the bounds of the nebula and is spatially closely associated with the candidate clusters from the previous subsection. Indeed, eight of the radio sources are spatially coincident with candidate clusters identified in the previous section (and are listed in Table 1). The presence and spatial coincidence of significant quantities of ionised gas further strenghtens the proposal in Sect. 2 that the mid-IR emission originates in a photoionisation front on the surface of a molecular cloud(s).

While the limitations of the current published data precludes the identification and spectral classification of the exciting OB stars of the H II emission - with the sole exception of WR 48a - we list in Table 1 both the requisite ionising flux for each region and the corresponding number of "canonical" O7V stars required to power the regions, adopting the appropiate ionising fluxes from Smith et al. (2002). Note that these estimates assume neither photon leakage nor dust absorption; 
Table 2. Upper Panel: summary of the multiwavelength properties of the subset of IRAS sources with colours satisfying the classification criteria for ucHII regions of Wood \& Churchwell (1989). The bolometric luminosities given in Col. 2 are estimated from IRAS 12-100 $\mu$ m fluxes following the methodology of Walsh et al. (1997), while the spectral type of a putative single star required to power the source is given in Col. 3. Column 4 lists the possible host $\mathrm{H}$ II regions. Comparison of the positions of Caswell $\mathrm{CH}_{3} \mathrm{OH} \& \mathrm{H}_{2} 0305.20+00.21$ to the radio map presented by Danks et al. (1984) suggest that they lie on the periphery of the H II region [CH87] 305.254+0.204, while the radio source PMN J1308-6215 is offset by 27" from the IRAS source IRAS 13054-6159. Given the uncertainty in identifying the radio and IRAS/molecular sources with one another, in both cases the H II region in question is given in itallics. Finally Col. 5 lists associated molecular and maser emission and $\mathrm{ucH}$ II regions, for which the LyC ionising fluxes and spectral type inferred for the exciting star are given in parenthesis. Lower panel: presence of other maser and molecular emission spatially coincident with the H II regions that host the aforementioned IRAS sources. Radio positions are accurate to $1 \mathrm{arcsec}$ (Caswell \& Haynes 1987), SiO to $<3$ arcsec (Harju et al. 1998) and maser emission to $<5$ arcsec (Caswell et al. 1995) for Parkes detections and $\sim 0.5$ arcsec (Norris et al. 1993) for the subset of Australian Telescope Compact Array observations.

\begin{tabular}{|c|c|c|c|c|}
\hline $\begin{array}{l}\text { IRAS } \\
\text { Source }\end{array}$ & $\begin{array}{c}L_{\mathrm{IR}} \\
\left(10^{4} L_{\odot}\right) \\
\end{array}$ & $\begin{array}{l}\text { Spectral } \\
\text { type }\end{array}$ & $\begin{array}{l}\text { Host H II } \\
\text { region }\end{array}$ & $\begin{array}{l}\text { Spatially associated maser, } \\
\text { molecular \& ucHII source(s) }\end{array}$ \\
\hline IRAS 13054-6159 & 11.0 & O8.5 & $P M N J 1308-6215$ & [HLB98] SEST 58 (SiO) \\
\hline IRAS 13070-6227 & 6.2 & B0 & - & [HLB98] SEST 59 (SiO) \\
\hline IRAS 13079-6218 & 15.8 & O7.5 & [CH87] 305.254+0.204 & Caswell $\mathrm{CH}_{3} \mathrm{OH} \& \mathrm{H}_{2} 0305.20+00.21$ \\
\hline IRAS 13080-6229 & 26.9 & O5 & {$[\mathrm{CH} 87] 305.202+0.022$} & $\begin{array}{l}\text { Caswell } \mathrm{CH}_{3} \mathrm{OH} 305.20+00.02 \\
\text { Caswell } \mathrm{CH}_{3} \mathrm{OH} 305.20+00.019 \\
1 \times \text { ucHII }(\log N \text { LyC } \geq 47.2, \geq \mathrm{B} 0.5 \mathrm{~V})\end{array}$ \\
\hline IRAS 13092-6218 & 42.5 & $\mathrm{O} 3$ & [CH87] 305.363+0.179 & $\begin{array}{l}\text { Caswell } \mathrm{H}_{2} 0305.36+00.20 \\
\text { [HLB98] SEST } 61(\mathrm{SiO}) \\
1 \times \text { ucHII }(\log N \text { LyC }=47.1, \mathrm{~B} 0.5 \mathrm{~V})\end{array}$ \\
\hline IRAS 13107-6208 & 5.5 & B0 & {$[\mathrm{CH} 87] 305.537+0.338$} & - \\
\hline IRAS 131111-6228 & 13.7 & O8 & [CH87] 305.551-0.005 & $\begin{array}{l}2 \times \text { ucHII } \\
(\log N \text { LyC } \geq 46.0, \geq \mathrm{B} 1.5 \mathrm{~V}) \\
(\log N \text { LyC }=45.8,<\mathrm{B} 1.5 \mathrm{~V})\end{array}$ \\
\hline \multirow[t]{4}{*}{ IRAS 13134-6242 } & 6.2 & B0 & - & $\begin{array}{l}\text { Caswell } \mathrm{CH}_{3} \mathrm{OH} \text { and } \mathrm{H}_{2} 0305.80-00.24 \\
1 \times \text { ucHII }(\log N \text { LyC }=45.5,<\mathrm{B} 1.5 \mathrm{~V})\end{array}$ \\
\hline & & & {$[\mathrm{CH} 87] 305.202+0.022$} & $\begin{array}{l}\text { Caswell } \mathrm{CH}_{3} \mathrm{OH} \text { and } \mathrm{OH} 305.21+00.02 \\
\text { Caswell } \mathrm{H}_{2} \mathrm{O} 305.199+00.005\end{array}$ \\
\hline & & & [CH87] 305.254+0.204 & $\begin{array}{l}\text { Caswell } \mathrm{CH}_{3} \mathrm{OH} 305.25+00.25 \\
\text { Caswell } \mathrm{OH} 305.208+00.206 \\
\text { Caswell } \mathrm{CH}_{3} \mathrm{OH} 305.21+00.21 \\
\text { [HLB98] SEST } 60(\mathrm{SiO})\end{array}$ \\
\hline & & & [CH87] 305.363+0.179 & $\begin{array}{l}\text { Caswell } \mathrm{CH}_{3} \mathrm{OH} 305.36+00.15 \\
\text { Caswell } \mathrm{OH} 305.362+00.150 \\
\text { Caswell } \mathrm{CH}_{3} \mathrm{OH} 305.37+00.19 \\
\text { Caswell } \mathrm{H}_{2} 0305.37+00.21\end{array}$ \\
\hline
\end{tabular}

in reality we consider it highly likely that one or both such assumptions will be violated. The implications of these estimates will be addressed in Sect. 4 .

\subsection{Ultra compact $\mathrm{H} \|$ regions ( $\mathrm{ucH} \|$ )}

Very young star-forming regions may be identified from IRAS observations by implementing the classification criterion for ultra-compact H II (ucH II) regions by Wood \& Churchwell (1989); $\log \left(f_{25} / f_{12}\right)>0.57$ and $\log \left(f_{60} / f_{12}\right)>1.3$. The regions so selected are listed in Table 2, four of which are found to be associated with ucH II regions catalogued by Walsh et al. (1998). The ucH II regions (see Fig. 2 and Table 2) show a close association with both the IR clusters, and the more extended host H II regions.
ucH II regions are thought to arise as a massive OB star ionises its immediate birth environment via its Lyman continuum flux. The Lyman continuum fluxes inferred from the radio fluxes for each of the ucH II regions are presented in Table 2, and are consistent with a population of early B stars (Smith et al. 2002). Since these estimates address neither possible photon leakage nor dust absorption, we consider them to be lower limits to the true spectral types of the embedded stars.

\subsection{Maser and molecular emission}

Maser and molecular emission often occur in conjunction with star forming regions. $\mathrm{H}_{2} \mathrm{O}$ and $\mathrm{OH}$ maser emission can arise 
from a variety of astrophysical objects ${ }^{3}$, although methanol maser emission is found to be closely correlated to highmass star-forming regions (Walsh et al. 1997). SiO emission is thought to occur behind shocks driven by energetic outflows from young stellar objects and hence form a diagnostic of star formation even in the absence of other signifiers (e.g. Harju et al. 1998). We have compiled $\mathrm{H}_{2} \mathrm{O}, \mathrm{OH}$ and methanol masers from Caswell (1998) and Caswell et al. (1989, 1995). SiO emission is catalogued by Harju et al. (1998) The positions of the molecular emission are marked in Fig. 2, and summarised in Table 2, along with their related positional uncertainties ${ }^{4}$.

Four of the eight IRAS regions selected in Sect. 3.3 are found to be associated with one or more $\mathrm{H}_{2} \mathrm{O}, \mathrm{OH}$ and/or methanol masers. Several masers apparently associated with IRAS 13079-6218 lie on the periphery of the H II region [CH87] 305.254+0.204, while additional maser emission is also found within the confines of [CH87] 305.254+0.204 and $305.363+0.179$ (Table 2; lower panel). Finally $\mathrm{SiO}$ emission is associated with four of the IRAS sources and [CH87] $305.254+0.204$. We therefore conclude that significant, ongoing massive star formation is occuring at these locations.

We may use the integrated IR fluxes to determine the bolometric luminosities for the embedded, exciting star(s). Unfortunately, due to the crowded nature of the field, source confusion and contamination will clearly lead to overestimates for the bolometric luminosity of "individual" sources (e.g. Crowther \& Conti 2003), even with the higher spatial resolution afforded by MSX. Indeed, high spatial resolution $\left(<3^{\prime \prime}\right)$ mid-IR imaging of the regions centred on IRAS 13070-6227 and 13079-6218 revealed that the spatially extended sources observed in the MSX data are resolved into several distinct objects in both cases (Walsh et al. 2001). Moreover, utilising mid-IR fluxes alone to infer the bolometric luminosity of an embedded source requires a number of assumptions as to the temperature(s), geometry and composition of the radiating dust to be made. An example of the dangers of such an approach can be seen from the extrapolation of a bolometric luminosity from the 10 micron flux of source 27 of Walsh et al. (2001; one of 2 components of IRAS 13079-6227), where a value an order of magnitude in excess of that determined from the IRAS fluxes was obtained.

Therefore, in Table 2 we simply choose to present bolometric luminosities determined from IRAS fluxes via Eq. (3) of Walsh et al. (1997), noting that due to the inevitable source confusion/blending we consider these estimates to be upper limits for the true bolometric luminosity of each - likely composite - source. Despite this caveat, it is clear that the formation of a population of deeply embedded OB stars is currently occuring at a number of locations on the periphery of the

\footnotetext{
${ }^{3}$ Note that the higher resolution observations of Walsh et al. (2001) do not confirm the association of Caswell $\mathrm{H}_{2} \mathrm{O} 305.20+00.21$ with the foreground M supergiant IRS 7 as suggested by Danks et al. (1984); likewise Caswell $\mathrm{H}_{2} \mathrm{O} 305.36+00.20$ is $>80^{\prime \prime}$ from the similar foreground M star IRS 42 (notation for both sources from Danks et al.).

${ }^{4}$ We have assumed coincidence if the positions agree to within $\pm 1 \sigma$ of the absolute IRAS position, noting that the accuray of the IRAS position is dependant on source flux and is in general better than 20 arcsec (Beichman et al. 1988).
}

nebula - supporting the conclusions drawn from the ucH II fluxes - with spectral types for putative single exciting stars ranging from $\mathrm{O} 3-\mathrm{B} 0$.

\section{Discussion}

\subsection{Distance to the complex}

Based on the optical-near IR colours, Danks et al. (1983) suggest a distance and reddening for WR 48a of 3.7-4.5 kpc and $A_{\mathrm{v}} \sim 9.2$ mag respectively, consistent with an origin in either Danks 1 or 2 . Given an average value of $A_{\mathrm{v}}=1.8 \mathrm{mag} / \mathrm{kpc}$ (Whittet 1992) and an abrupt increase in $A_{\mathrm{v}}=0.7$ to $4.0 \mathrm{mag}$ at $1 \mathrm{kpc}$ (Neckel \& Klare 1980) such a combination of values is internally consistent. A kinematic distance estimate of 3.5-4 kpc for the surrounding star forming complex has been determined from a combination of radio and $\mathrm{H} \alpha$ observations (e.g. Georgelin et al. 1988; Russeil et al. 1998). While consistent with the distance estimate obtained for WR 48a, Russeil et al. (1998) caution that large velocity dispersions are present in the $\mathrm{H} \alpha$ data - presumably due to significant internal cloud motions - which complicates the determination of a unique distance to the G305 complex.

For this paper, we have therefore adopted a distance of $4 \mathrm{kpc}$ for WR 48a, the optical clusters Danks 1 and 2 - separated from one another by only $3 \mathrm{pc}$ at this distance - and the surrounding star forming complex, noting that the kinematic data suggests this forms an upper limit to both distance and luminosities for the exciting sources ${ }^{5}$.

\subsection{Age estimates of WR 48a, and Danks 1 and 2}

At the above distance and reddening we find a luminosity for WR 48a of $\log \left(L / L_{\odot}\right) \sim 5.6$, utilising a bolometric correction of -4 mag in the $V$ band (determined from modeling of the spectra of the WC8 stars WR 57 and 60; P. Crowther, priv. comm. 2003). Following the prescription for hydrogen free WRs of Schaerer \& Maeder (1992) we infer a present mass for WR $48 \mathrm{a}$ of $\sim 17 M_{\odot}$, which, given the unknown contribution from a putative binary companion (dust producing WRs are most often binary systems, e.g.Williams \& van der Hucht 2000 and references therein) we adopt as an upper limit. Such a mass estimate suggests a rather massive progenitor for WR 48a, and hence an age for both star and the clusters Danks 1 and 2 at the lower end of the range for the onset of the WC phase of 2.5-3.7 Myr (depending on initial mass, Maeder \& Meynet 1994).

\footnotetext{
5 Given the depth of the observations by Danks et al. (1984) it seem highly likely that the true radial extent of the clusters is underestimated and hence, with an angular displacement of only 1 arcmin from either cluster, WR 48a could well be located in a putative cluster "halo". Alternatively, assuming a lifetime of $3 \mathrm{Myr}$ for WR 48a, a velocity of only $1 \mathrm{~km} \mathrm{~s}^{-1}$ is required for it to have been dynamically ejected from either cluster, entirely consistent with the observed velocity distribution of OB runaways; hence we assume an origin of WR 48a in either Danks 1 or 2.
} 
The lack of luminous red supergiants (which would have dominated the near-IR output of Danks 1 and 2 if present) suggests an upper limit to the age of the cluster of $\sim 4-5 \mathrm{Myr}$ - no stars of $\sim 30 M_{\odot}$ or less have evolved from the main sequence.

Danks et al. (1984) reports an integrated magnitude of the cluster Danks 2 of $K \sim 5.3^{6}$, (no photometry of individual cluster members exists) corresponding to a reddening corrected $M_{K} \sim-8.4$. The absolute $K$ band magnitude of O3-B0 supergiants are in the range of $M_{K}=-5.6--5.9$ (Clark et al. 2004; based on detailed NLTE modeling). Therefore it is likely that this cluster contains a significant population of luminous early type stars.

However, the brightest stars are spatially resolved in Danks 1 , and were found to have $K$ band magnitudes of $\sim 8$ mag implying $M_{K} \approx-6$. This value exceeds that of a single main sequence O star (Blum et al. 2000), implying that a population of luminous evolved stars must also be present in this cluster. This supposition is supported by the limited spectroscopy of cluster members of Danks et al. (1984), which show both Paschen lines (indicative of an $\mathrm{O}-\mathrm{A}$ classification) and $\mathrm{H} \alpha$ emission for one star, likely due to the presence of a powerful stellar wind.

Alternative explanations for the brightest resolved stars, such as identification as classical Be stars or the presence of excess near-IR emission from a surviving natal disc may be rejected. The earliest spectral types for which the Be phenomenon is observed are late $\mathrm{O}$ stars, which would have $M_{K} \sim$ -5 , a magnitude fainter than the cluster members in question (Porter 2003). Likewise assuming an age for Danks 1 in excess of $3 \mathrm{Myr}$, it is highly likely that a dusty natal disc will have dispersed. From Eq. (4) of Shepherd, Kurtz \& Testi (2004) and assuming the maximum gravitationally stable mass for an accretion disc $\left(0.3 M_{*}\right.$; Shu et al. 1990) we find that a putative natal accretion disc around a $20 M_{\odot}$ (early B) star would have a maximum lifetime of $\leq 1 \mathrm{Myr}$ - with similar discs around more massive stars having shorter lifetimes - significantly less than the age of the cluster.

\subsection{Star formation in the periphery of the nebula}

At a distance of $4 \mathrm{kpc}$, the projected maximal extent of the nebula is $\sim 30 \mathrm{pc}$, although the projected distances of the embedded sources - IR clusters, masers and ucH II regions - from Danks 1 and 2 range from $\sim 4-25$ pc ([DBSB2003] 132 and 133 respectively). These are predominantly located in the narrow $(\sim 2 \mathrm{pc})$ photodissociation region on the boundary of the nebula. For a typical sound speed of $10 \mathrm{~km} \mathrm{~s}^{-1}$, this corresponds to a crossing time of only $10^{5} \mathrm{yr}$.

While an evolutionary paradigm for massive stars has yet to be fully constructed, Evans et al. (2002) and Crowther \& Conti (2003) outline the observational features that such a theory must accomodate. At the earliest stages, maser emission, possibly arising in a circumstellar disc or in shocks behind molecular outflows is observed, while the driving source may be

\footnotetext{
${ }^{6}$ We choose to utilise near-IR magnitudes since this will minimise the effect of possible spatially inhomogeneous reddening across the clusters, as suspected by Danks et al. (1984).
}

identified in the IR via heating of the dust envelope. H II emission is not observed, due either to insufficient production of LyC photons or to the rate of accretion being high enough to quench such emission. As one or both of these conditions is violated the star develops an ucH II region, a phase which is expected to last for some $\sim 10^{5} \mathrm{yr}$. Finally, the ucH II regions expands as the natal envelope disperses, accompanied by the emergence of the star at near-IR and finally optical wavelengths.

The morphology of the nebula, together with the nature and location of the embedded sources is therefore strongly suggestive of the occurence of star formation on the periphery of the cavities traced by the MSX emission. We suggest this to have been triggered by the sweeping up of natal gas by the winds and radiation field of the hot star component of Danks 1 and 2 ; indeed, the complex fulfills both of the morphological signatures for triggered star formation suggested by Elmegreen (1992) which were cited in Sect. 1.

In this respect, the location of PMN J1308-6215 is of particular interest, since the colours of the associated IRAS source 13054-6159 and the presence of $\mathrm{SiO}$ emission implies ongoing star formation occuring in isolation from the main complex. Such a conclusion is supported by the near equivalence of the luminosity of the exciting source - a single late $\mathrm{O}$ main sequence star - determined from both radio and IR fluxes (Tables 1 and 2), implying that no external source of energy is required to power the nebula. Under the assumption that it is at a distance of $4 \mathrm{kpc}$ we find a radius of $\sim 1.2 \mathrm{pc}$ and therefore can utilise the results of Dyson \& Williams (1980) to determine an age of $\sim 1.7 \times 10^{5} \mathrm{yr}$ (assuming the bubble expands into a medium of uniform $10^{3} \mathrm{~cm}^{-3}$ density). Given the apparent youth of the feature, its projected distance and isolation from the main complex, we speculate that high mass star formation is not limited to that region alone, but instead is ongoing and multiseeded through the host molecular cloud.

\subsection{Comparison with other star forming regions}

Given both the youth, relative proximity and inferred stellar content - in excess of 31 canonical O stars - for the complex, it is instructive to compare it to other known regions of massive star formation in the galaxy. Morphologically, the complex appears similar to the Rosette nebula at mid-IR wavelengths (e.g. Kraemer et al. 2003). Both nebulae are likely to have been formed by the interaction of the central cluster(s) of OB stars (NGC 2244 in the case of the Rosette nebula). Phelps \& Lada (1997) also report the detection of a number of embedded compact clusters which they suggest result from the interaction of NGC 2244 with the host molecular cloud. Embedded cluster are also found in the environs of the young open clusters IC 1396 and IC 1805 (Clarke et al. 2000 and references therein), although the hot star population inferred for the G305 complex is significantly in excess of the populations of any of these regions.

Comparison with the compilation of the mid-IR and radio properties of galactic giant $\mathrm{H}$ II regions presented by Conti \& Crowther (2004) suggests that G305 complex is amongst the 
most massive star forming regions in the galaxy. Such a conclusion is further supported by consideration of the dispersal of the natal molecular cloud surrounding Danks 1 and 2 over their adopted lifespan of $\sim 3 \mathrm{Myr}$, thus substantially reducing the radio and IR luminosity of the complex. Natal cloud clearing was suggested to explain the unexpectedly small mid IR and radio luminosity of the $\sim 10^{5} M_{\odot}$ starburst cluster Westerlund 1 (Clark et al. 2004, in prep.), when compared to radio/IR bright regions such as W49 (which Alves \& Homeier 2003 show to host $\sim 100$ deeply embedded $\mathrm{O}$ star candidates). We speculate that given the morphology of the G305 complex and in particular the fact that sufficient natal material has been dispersed to allow Danks 1 and 2 to become optically visible, there is a likelihood of substantial photon leakage and thus we may be significantly underestimating the massive star population of the complex.

\section{Conclusion}

Mid-IR observations of the $\sim 1.5^{\circ} \times 1.5^{\circ}$ region centered on WR 48a reveals a spectacular 3 lobed nebula. The overall morphology is suggestive of a wind blown structure powered by the WR 48a and the hot stellar component of the nearby clusters Danks 1 and 2. Given the proximity of WR 48a to the optical clusters Danks 1 and 2 a common origin is suggested and a minimum age of $\geq 3$ Myrs estimated. The apparent lack of red supergiants within both clusters suggests an upper limit to their ages, and by extension the nebula, of $\sim 4-5 \mathrm{Myr}$.

Analysis of 2MASS data by Dutra et al. (2003) indicates the presence of a population of clusters spatially coincident with the mid-IR hotspots present on the periphery of the nebula. A search for molecular emission at these locations reveals numerous sites of $\mathrm{H}_{2} \mathrm{O}, \mathrm{OH}$ and methanol maser emission. From these observations we identify at least two different generations of star formation within the G305 radio complex. The earliest stars to form we associate with the optically visible clusters Danks 1 and 2, and possible outlying cluster members such as WR48a. Secondly, the emdedded IR sources, maser emission and $\mathrm{ucH}$ II regions herald a population of early OB stars still in the process of formation.

The overal morphology of the complex is therefore consistent with numerous sites of ongoing massive OB star formation, which have apparently been triggered by an interaction of the parent molecular cloud(s) with the winds and ionising radiation of the hot star population of the star clusters Danks 1 and 2. However, we note at least one location of apparently isolated massive star formation located away from the main complex suggesting that a multiseeded component of massive star formation may also be present.

Significant radio emission is found to be coincident with the periphery of the mid-IR nebula. The radio flux implies a minimum population of 31 canonical $\mathrm{O} 7 \mathrm{~V}$ stars is required to provide a sufficient LyC ionising flux. However, given the likelihood of significant photon leakage we suspect that such a value is likely to be a significant underestimate. As such, this complex likely forms one of the most massive regions of ongoing star formation currently identified within the galaxy.
Acknowledgements. We thank Mike Egan, Paul Crowther, Peter Conti, Martin Cohen \& Simon Goodwin for many useful discussions. This research made use of data products from the Midcourse Space Experiment. Processing of the data was funded by the Ballistic Missile Defense Organisation with additional support from NASA Office of Space Science. This research has also made use of the NASA/IPAC Infrared Science Archive, which is operated by the Jet Propulsion Laboratory, California Institute of Technology, under contract with the National Aeronautics and Space Administration.

\section{References}

Alves, J., \& Homeier, N. 2003, ApJ, 589, L45

Beichman, C. A., Neugebauer, G., Habing, H. J., Clegg, P. E., \& Chester, T. J. 1988, NASA RP-1190, Vol. 1

Blum, R. D., Conti, P. S., \& Damineli, A. 2000, AJ, 119, 1860

Caswell, J. L. 1998, MNRAS, 297, 215

Caswell, J. L., \& Haynes, R. F. 1987, A\&A, 171, 261

Caswell, J. L., Batchelor, R. A., Forster, J. R., \& Wellington, K. J. 1989, AuJPh, 42, 331

Caswell, J. L., Vaile, R. A., Ellingsen, S. P., et al. 1995, MNRAS, 272, 96

Clark, J. S., Negueruela, I., Crowther, P., \& Goodwin, S. 2004, A\&A, in prep.

Clarke, C. J., Bonnell, I. A., \& Hillenbrand, L. A. 2000, in Protostars and Planets IV, ed. V. Mannings, A. P. Boss, \& S. S. Russell (Book - Tucson: University of Arizona Press), 151

Conti, P. S., \& Crowther, P. A. 2004, MNRAS, in press [arXiv:astro-ph/0307365]

Crowther, P. A., \& Conti, P. S. 2003, MNRAS, 343, 143

Cutri, R. M., Skrutskie, M. F., Van Dyk, S., et al. 2003, Explanatory Supplement to the 2MASS All Sky Data Release

Danks, A. C., Dennefeld, M., Wamsteker, W., \& Sahver, P. A. 1983, A\&A, 118, 301

Danks, A. C., Wamsteker, W., Shaver, P. A., \& Retallack, D. S. 1984, A\&A, 132, 301

Dutra, C. M., Bica, E., Soares, J., \& Barbury, B. 2003, A\&A, 400, 533

Dyson, J. E., \& Williams, D. A. 1980, The Physics of the Interstellar Medium (Manchester: Manchester Univ. Press)

Egan, M. P., et al. 2003, The Midcourse Space Experiment Point Source Catalogue Version 2.3 Explanatory Guide

Elmegreen, B. G. 1992, in Star Formation in Stellar Systems, ed. G. Tenorio-Tagle, M. Prieto \& F. Sanchez (Cambridge: Cambridge Univ. Press), 381

Elmegreen, B. G., \& Lada, C. J. 1977, ApJ, 214, 725

Evans II, N. J., Shirley, Y. L., Mueller, K. E., \& Knez, C. 2002, in Hot Star Workshop III: The Earliest Phases of Massive star Birth, ASP Conf. Ser., ed. P. A. Crowther

Goss, W. M., \& Shaver, P. A. 1970, AuJPA, 14

Georgelin, Y. M., Boulesteix, J., Georgelin, Y. P., et al. 1988, A\&A, 205, 95

Harju, J., Lehtinen, K., Booth, R. S., \& Zinchenko, I. 1998, A\&AS, 132, 211

Kraemer, K. E., Shipman, R. F., Price, S. D., et al. 2003, AJ, 126, 1423

Maeder, A., \& Meynet, G. 1994, A\&A, 287, 803

McKee, C. F., \& Tan, J. C. 2002, Nature, 416, 59

Mill, J. M., O’Neil, R., Price, S. D., et al. 1994, JSpRo, 31, 900

Neckel T., \& Klare G. 1980, A\&AS, 42, 251

Norris, R. P., Whiteoak, J. B., Caswell, J. L., et al. 1993, ApJ, 412, 222

Phelps, R. L., \& Lada, E. A. 1997, ApJ, 477, 176

Porter, J. M. 2003, Be Star Newsl., 36, 6 
Price, S. D., Egan, M. P., Carey, S. J., et al. 2001, AJ, 121, 2819

Russeil, D., Georgelin, Y. M., Amram, P., et al. 1998, A\&AS, 130, 119

Schaerer, D., \& Maeder, A. 1992, A\&A, 263, 129

Shepherd, D. S., Kurtz, S. E., \& Testi L. 2004, ApJ, 601, 952

Shu, F., Tremaine, S., Adams, F., \& Ruden, S. 1990, ApJ, 358, 495

Smith, L. J., Norris, R. P. F., \& Crowther, P. A. 2002, MNRAS, 337, 1309

van der Hucht, K. A. 2001, NewAR, 45, 2001

Walsh, A. J., Hyland, A. R., Robinson, G., \& Burton, M. G. 1997, MNRAS, 291, 261
Walsh, A. J., Burton, M. G., Hyland, A. R., \& Robinson, G. 1998, MNRAS, 301, 640

Walsh, A. J., Bertoldi, F., Burton, M. G., \& Nikola, T. 2001, MNRAS, 326, 36

Whittet, D. C. B. 1992, Dust in the Galactic Environment (Bristol: Inst. Phys.)

Williams, P. M., \& van der Hucht, K. A. 2000, MNRAS, 314, 23

Wood, D. O. S., \& Churchwell, E. 1989, ApJ, 340, 265

Wright, A. E., Griffith, M. R., Burke, B. F., \& Ekers, R. D. 1994, ApJS, 91,111 